\title{
Úlceras por presión en el paciente hospitalizado
}

\author{
Pressure ulcers in the hospitalized patient
}

\section{Señor Editor:}

La prevalencia de úlceras por presión (UPP) en pacientes hospitalizados varía entre el 5 y $15 \%$, siendo mayor en pacientes admitidos a unidades de cuidados intensivos (UCI) y servicios de larga estancia ${ }^{(1)}$. A pesar de los esfuerzos realizados en años recientes para su diagnóstico y tratamiento oportuno, constituyen en la actualidad un serio problema de salud pública, con consecuencias físicas, psicológicas y sociales, que afectan la calidad de vida de los pacientes ${ }^{(2,3)}$.

En el estudio publicado por Flores-Lara Y. y col. (4), se estableció como objetivo determinar la frecuencia de UPP y los factores asociados a su presentación, en pacientes de un hospital nacional de Lima, Perú. Para ello, los autores reportan el número de pacientes con UPP por semanas de hospitalización, atendidos en diferentes servicios de hospitalización durante el año 2016. Durante dicho periodo se registraron 30 casos de UPP, siendo la mayoría observados en pacientes de UCI (41,67\%), Cirugía General (16,67\%) y Medicina Interna $(9,52 \%)$. Además, el $60 \%$ del total de casos de UPP se presentaron en adultos mayores de 60 años.

Sin embargo, el análisis del artículo permite identificar una serie de limitaciones metodológicas que los autores no comentan en la discusión y que influyen en la validez interna y externa de los resultados obtenidos, como son: el diseño y objetivos del estudio, la delimitación de la población y la definición de casos.

La frecuencia de UPP por cada servicio del hospital se determinó en base al total de casos observados durante el año 2016, sin considerar el número de pacientes hospitalizados durante dicho periodo. La determinación de factores asociados a la presentación de UPP requiere de un diseño de tipo analítico y no descriptivo, que los autores pasan por alto, reportando únicamente diferencias estadísticamente significativas en el número de casos por servicio de hospitalización y la distribución de los casos por grupos de edad ${ }^{(5)}$. Al respecto, los factores asociados a las UPP pueden diferir cuando se estudian pacientes de UCI, Cirugía General, Medicina Interna y Obstetricia, como consecuencia de la edad, motivo de hospitalización, comorbilidades, condición nutricional, estado funcional, uso de fármacos, tiempo de estancia hospitalaria, así como de la adherencia por parte del personal sanitario a los protocolos de prevención y manejo de esta patología (2).

Finalmente, el estudio no aclara la definición empleada para el diagnóstico de UPP, el grado de las lesiones que va del grado I (eritema que no desaparece a la digitopresión) al IV (úlcera profunda que compromete la fascia muscular y tejidos subyacentes), la descripción de pacientes con UPP múltiples, ni diferencia los casos presentes al momento del ingreso de aquellos que aparecieron durante la hospitalización, lo cual compromete de manera significativa la interpretación de los resultados ${ }^{(4)}$.

El desarrollo de UPP durante la hospitalización constituye un indicador de la calidad de atención en salud. En un estudio descriptivo realizado con registros electrónicos de 3904 pacientes hospitalizados $(66 \%$ adultos mayores de 65 años), 962 (24,6\%) presentaron un alto riesgo de UPP al momento de la admisión hospitalaria. De manera interesante, 3458 (88,6\%) no tuvieron una inspección visual de la piel en busca de lesiones, evidenciándose inconsistencias entre los reportes de médicos y enfermeros ${ }^{(6)}$.

Facultad de Medicina Humana "Manuel Huamán Guerrero". Universidad Ricardo Palma. Lima, Perú.

Hospital Nacional Hipólito Unanue. Lima, Perú.

a Médico geriatra. 
Estos datos refuerzan la necesidad de un adecuado sistema de monitoreo de UPP en el paciente hospitalizado, con énfasis en la población vulnerable, especialmente el grupo de adultos mayores. El sistema debería acompañarse de un programa de prevención, que incluya la educación del personal de salud, pacientes y familiares; así como el seguimiento de los pacientes por parte de un equipo multidisciplinario ${ }^{(7)}$.

\section{Jaime Lama ${ }^{1,2, a}$, Rodrigo Rodríguez ${ }^{1,2, a}$}

\section{Correspondencia:}

Jaime Enrique Lama Valdivia

Calle Pirandello 488, Dpto 201, Urb. San Borja.

Lima 41, Perú.

Teléfono: 051999093383

Correo electrónico: jlamav@hotmail.com

\section{REFERENCIAS BIBLIOGRÁFICAS}

1. Vangilder C, Macfarlane GD, Meyer S. Results of nine international pressure ulcer prevalence surveys: 1989 to 2005. Ostomy Wound Manage. $2008 \mathrm{Feb}$; 54(2):4054. PMID: 18382042.

2. Mervis JS, Phillips TJ. Pressure ulcers: Pathophysiology, epidemiology, risk factors, and presentation. J Am Acad Dermatol. 2019 Oct; 81(4):881-890. doi: 10.1016/j.jaad.2018.12.069. Epub 2019 Jan 18. PMID: 30664905.

3. Gorecki C, Brown JM, Nelson EA, Briggs M, Schoonhoven L, Dealey C, Defloor T, Nixon J; European Quality of Life Pressure Ulcer Project group. Impact of pressure ulcers on quality of life in older patients: a systematic review. J Am Geriatr Soc. 2009 Jul; 57(7):1175-83. doi: 10.1111/j.15325415.2009.02307. x. Epub 2009 May 21. PMID: 19486198.

4. Flores-Lara Y, Rojas-Jaimes J, Jurado-Rosales J. Frecuencia de úlceras por presión y los factores asociados a su presentación, en pacientes de un hospital nacional de Lima, Perú. RMH [Internet]. 16oct.2020 [Citado el 2 noviembre de 2020]; 31(3):164-8. Disponible en: https://revistas.upch.edu. pe/index.php/RMH/article/view/3805

5. Grimes DA, Schulz KF. Descriptive studies: what they can and cannot do. Lancet. 2002 Jan 12; 359(9301):145-9. doi: 10.1016/S0140-6736(02)073 73-7. PMID: 11809274.

6. Gaspar S, Collier M, Marques A, Ferreira C, Gaspar de Matos M. Pressure ulcers: The challenge of monitoring in hospital context. Appl Nurs Res. 2020 Jun; 53:151266. doi: 10.1016/j.apnr.2020.151266. Epub 2020 Apr 11. PMID: 32451008.
7. Al Mutair A, Ambani Z, Al Obaidan F, Al Salman K, Alhassan H, Al Mutairi A. The effectiveness of pressure ulcer prevention programme: A comparative study. Int Wound J. 2020 Feb; 17(1):214-219. doi: 10.1111/iwj.13259. Epub 2019 Nov 6. PMID: 316 96665.

Recibido: 03/11/2020

\section{Réplica de los autores}

\section{Authors' reply}

\section{Sr. Editor}

El objetivo del estudio fue determinar la frecuencia de úlceras por presión y los factores asociados a su presentación, en pacientes de un hospital nacional de Lima, Perú, y se describieron las limitaciones en el artículo.

Solo se realizó la prueba de Chi cuadrado como estadística de asociación entre servicios y UPP, teniendo los servicios por las características de ingreso para los pacientes ciertas condiciones porque nos pareció importante la asociación y que al ser dos variables tenemos una relación estadística analítica. Como bien indican los autores de la carta al editor, otros factores como comorbilidades, condición nutricional, uso de fármacos, tiempo de estancia y otros son variables independientes que definitivamente repercuten en la presentación de los casos y no fueron tomadas en cuenta declarando estas en el marco de las limitaciones.

Finalmente, la definición de UPP es una nomenclatura ya conocida por lo que no fue necesario detallar esta, respecto a la discriminación del tiempo de cuando apareció la UPP, es correcta la apreciación de indicar como un vacío de nuestro estudio y limitación de esta.

Agradecemos mucho las observaciones en bien de nuestra mejora como investigadores.

\section{Jesús Rojas-Jaimes ${ }^{1, a}$}

\footnotetext{
Facultad de Salud, Universidad Privada del Norte. Lima, Perú.

2 Magister en Biología Molecular.
} 